\begin{tabular}{|c|c|}
\hline Title & Macrosegregation simulation model based on Lattice Boltzmann method with high computational efficiency \\
\hline Author(s) & Ohno, Munekazu; Sato, Hay ato \\
\hline Citation & $\begin{array}{l}\text { International journal of heat and mass transfer, } 127,561-570 \\
\text { https://doi.org/10.1016/.ijheatmasstransfer.2018.07.076 }\end{array}$ \\
\hline Issue Date & $2018-12$ \\
\hline DOC URL & http:/hdl.handle.net/2115/79860 \\
\hline Rights & $\begin{array}{l}\text { O2018. I his manuscript version is made avallabole under the CC-BY-NC-ND } 4.0 \text { IIcense } \\
\text { http://treativecommons.org/icenses/by-nc-nd/4.0/ }\end{array}$ \\
\hline Rights(URL) & http:/creativecommons.org/icenses/by-nc-nd/4.0/ \\
\hline Type & article (author version) \\
\hline File Information & 20180hno_IJHMT_HUSCAP.pdf \\
\hline
\end{tabular}

Instructions for use 


\title{
Macrosegregation simulation model based on Lattice-Boltzmann Method with high computational efficiency
}

\author{
Munekazu Ohno ${ }^{1)}$ and Hayato Sato ${ }^{2)}$
}

1) Division of Materials Science and Engineering, Faculty of Engineering, Hokkaido University, Kita 13Nishi 8, Kita-ku, Sapporo, Hokkaido 060-8628, Japan

2) Graduate Student, Graduate School of Engineering, Hokkaido University, Kita 13 Nishi 8, Kita-ku, Sapporo, Hokkaido 060-8628, Japan

\begin{abstract}
:
A macrosegregation simulation model is developed by coupling solute and energy conservation equations with Lattice-Boltzmann Method (LBM), newly developing technique of computational fluid dynamics. Effect of the solidification shrinkage is taken into account in the present LBM as well as effects of the Darcy's flow and thermos-solutal convection. The present LBM-coupled model is based on modified lattice Bhadnager-Gross-Krook method, the numerical stability of which is better than that of the standard LBM. Accordingly, the present LBM-coupled model can be applied to simulations of macrosegregation behaviors in metallic alloy systems that cannot be handled by the previous LBM-coupled model. The validity of the model was demonstrated by comparing the results for steady-state flows with those of analytical solutions and a conventional model based on the Navier-Stokes equation. In addition, the computational speed of the present model is compared with the one of conventional model in cases of lateral directional solidification of Sn-Bi alloy and continuous casting of a steel slab. It was shown that the present LBM-coupled model enables remarkably faster computation than the conventional model especially in the latter case.
\end{abstract}

Keywords:

Macrosegregation; Lattice-Boltzmann Method; Continuous casting; Ingot casting; Simulation 


\section{Introduction}

Macrosegregation refers to a non-uniform distribution of alloying elements on a scale of ingot. It stems from macroscopic flows of segregated liquids [1-4]. The macroscopic flow occurs in several mechanisms such as thermosolutal convection, a flow due to solidification shrinkage and forced flow and so on. Numerical models have been developed for simulating macrosegregation behaviors. The simulation models have been gradually sophisticated and their usefulness accordingly increases [5-7]. However, the macrosegregation simulations, in general, are computationally demanding and the high computational cost restricts the simulation system to a small size and/or it forces one to employ coarse gird, which often causes inaccurate description of macrosegregation behavior [4]. The numerical models are generally based on momentum, mass, energy and solute conservation equations where the momentum conservation is described by the Navier-Stokes (hereafter abbreviated as NS) equation. The numerical calculation for the NS equation requires a time-consuming calculation for correction of the velocity and pressure, which is a bottleneck restricting the speed-up of macrosegregation simulation. It is necessary to accelerate the calculation of fluid dynamics.

Lattice-Boltzmann Method (LBM) is a newly developing technique for computational fluid dynamics [8-14]. This is a method based on the lattice-Boltzmann equation that describes the time evolution of particle distribution functions, from which the macroscopic quantities of fluid such as the density, velocity and pressure can be calculated. Importantly, LBM does not require the time-consuming calculation for correction of the velocity and pressure and, hence, it generally enables fast computation of fluid dynamics. Recently, we developed a model for simulating macrosegregation based on LBM in which the NS equation is replaced by the lattice-Boltzmann equation including effects of the thermossolutal flow and Darcy's flow [15]. The accuracy of the LBM-coupled model was investigated by comparing the calculated results for the steady-state flows with the results obtained by analytical solutions and a conventional model based on the NS equation (NS-based model). The results of the LBM-coupled model are almost identical to those of the analytical and the NS-based models. Moreover, we conducted a simulation for solidification in a small ingot of a model alloy where the macrosegregation appears only by natural convection, by means of the LBM-coupled model and the NS-based model. The LBM-coupled model yields almost the same result as the NS-based model. Importantly, the simulation of LBM-coupled model is about five times faster than the one of NS-based model [15].

As described above, the acceleration of macrosegregation simulation was 
successfully achieved based on LBM. However, the following issues remain to be resolved. Although one cannot neglect effect of the macroscopic flow driven by the solidification shrinkage on the formation of macrosegregation in some cases [1-4], the solidification shrinkage cannot be dealt with in the previous model. More importantly, the LBM-coupled model developed in the previous study cannot be applied to real metallic alloy systems. In this model, the numerical stabilities of LBM and the energy conservation equation limit the Prandtl number to about one order of magnitude larger than typical values in metallic alloys [15]. In other words, when the typical value of thermal diffusivity is employed, the viscosity must be one order of magnitude higher than the typical value in metallic alloys in the light of the numerical stability of LBM. This problem must be resolved. Moreover, it is important to clarify how much acceleration can be achieved in simulations for realistic casting processes. The advantage of parallel computing technique should be investigated because LBM is suitable for parallel computing. These issues are tackled in this paper.

The purpose of this study is to construct a model for macrosegregation simulations with high computational efficiency based on LBM that can be applied to the simulations for realistic solidification processes in metallic alloy systems. To this end, the effect of solidification shrinkage is introduced in LBM. Moreover, the model is constructed based on the recently-developed Modified Lattice Bhadnager-Gross-Krook (MLBGK) method [16], the numerical stability of which is much higher than that of the standard LBM. Then, the model is applied to the simulations for lateral directional solidification of Sn-Bi alloy and continuous casting of steel. The computational speeds are investigated in comparison with the results of the conventional NS-based model. The paper is organized as follows. The LBM-coupled model and the NS-based model are explained in the next section. The results of steady-state flow, lateral directional solidification and continuous casting are described in sections 3, 4 and 5, respectively. The conclusions are given in the last section.

\section{Numerical methods}

\subsection{Conventional model based on the Navier-Stokes equation}

In this study, the LBM-coupled model is constructed by coupling the MLBK method with the solute and energy conservation equations. As is similar to the previous work, the present modeling rests on a NS-based model developed in early studies [17-19], which was chosen because the numerical implementation is relatively straightforward and the simulation results for lateral directional solidification of Sn-Bi alloy and continuous casting of steel were already reported [17-19]. 
In the NS-based model, the incompressible fluid flow is calculated by the following equations [19],

$$
\begin{aligned}
& \nabla \cdot \mathbf{u}=-\beta \frac{\partial f_{s}}{\partial t} \quad(1) \\
& \frac{\partial \mathbf{u}}{\partial t}+\mathbf{u} \cdot \nabla \mathbf{u}=-\frac{\nabla p}{\rho_{0}}+v \nabla^{2} \mathbf{u}+\frac{\rho_{0}+\delta \rho}{\rho_{0}} \mathbf{g}-\frac{v}{K} \mathbf{u}
\end{aligned}
$$

where $\mathbf{u}$ is the velocity vector of fluid, $\beta$ is the solidification shrinkage rate, $f_{s}$ is the volume fraction of solid, $p$ is the pressure, $\rho_{0}$ is the density at a reference state, $v$ is the kinematic viscosity, $\delta \rho$ represents the density change due to the variation of temperature and concentration, $\mathbf{g}$ is the gravitational acceleration and $K$ is the permeability. In contrast to the previous work, the continuity Eq. (1) includes the effect of solidification shrinkage. In the NS equation (Eq. (2)), the third and fourth terms on the right-hand side represent effects of thermosolutal convection and the Darcy's flow, respectively. In this study, the permeability $K$ is given by $K=K_{0}\left(1-f_{s}\right)^{3} / f_{s}{ }^{2}$, where $K_{0}$ is the permeability coefficient.

Equations (1) and (2) are coupled with the following energy conservation and solute conservation equations [17-19],

$$
\begin{aligned}
& \frac{\partial T}{\partial t}+\mathbf{u} \cdot \nabla T=a_{T} \nabla^{2} T+\frac{\Delta H}{C_{h}} \frac{\partial f_{s}}{\partial t} \\
& \frac{\partial C_{L}}{\partial t}+\mathbf{u} \cdot \nabla C_{L}=\frac{\partial f_{s}}{\partial t} \frac{\left(1-k_{e}\right) C_{L}}{1-f_{s}}
\end{aligned}
$$

where $T$ is the temperature, $a_{T}$ is the thermal diffusivity, $\Delta H$ is the latent heat and $c_{h}$ is the specific heat capacity, $C_{L}$ is the solute concentration in the liquid and $k_{e}$ is the equilibrium partition coefficient. In the solute conservation Eq. (4), the diffusion term is omitted and the reaction term on the right-hand side is based on the Scheil model.

Although several numerical methods were developed for solving the NS equation such as MAC [20] and SIMPLE methods [21], a time-consuming calculation is required for correction of the velocity and pressure in these methods. Hence, the computational burden for solving NS equation is generally high and it occupies a large part of the computational cost of macrosegregation simulations. Hence, a key to acceleration of the macrosegregation simulations lies in the calculation of fluid flow. In this study, the continuity Eq. (1) and NS Eq. (2) are replaced by LBM.

We carried out simulations of the above-mentioned NS-based model to compare its results with those of the LBM-coupled model. In the present study, Eqs. (1) and (2) were solved based on the SIMPLE method in all simulations. The energy and 
solute conservation Eqs. (3) and (4) were discretized based on a finite difference method with a second order accuracy in space and they were solved using a first order Euler scheme. One must pay attention to numerical accuracy of advection term because it affects the stability and accuracy of macrosegregation simulations. In this study, the advection terms were calculated based on the third-order upwind scheme. The total variation diminishing scheme [22] was employed to stabilize the numerical simulation only in the case of continuous casting of a steel slab.

\subsection{LBM-coupled model}

LBM has attracted a great deal of attention as an effective computational method for fluid dynamics [8-14]. LBM can be viewed as a discrete version of the Boltzmann equation. In the previous study, influence of solid fraction, Darcy's flow and thermosolutal convection are taken into account in LBM and it was coupled with the energy and solute conservation equations [15]. This LBM-coupled model developed in the previous work is first described in this sub-section. In this study, the model including the effect of solidification shrinkage is first developed based on the previous model, and then, it is reformulated based on the MLBGK model [16].

In LBM, the fluid consists of fictive and microscopic particles moving at discrete velocities in discrete directions on a lattice. Their collective behavior determines the macroscopic quantities of fluids such as the density, velocity and pressure. The lattice-Boltzmann equation is written as [14],

$$
f_{\alpha}\left(\mathbf{x}+\mathbf{e}_{\alpha} \delta t, t+\delta t\right)-f_{\alpha}(\mathbf{x}, t)=-\frac{1}{\tau}\left[f_{\alpha}(\mathbf{x}, t)-f_{\alpha}^{(e q)}(\mathbf{x}, t)\right]+F_{\alpha} \delta t
$$

where $f_{\alpha}$ represents the distribution function for the particle moving at a discrete velocity $\boldsymbol{e}_{\alpha}$ in a discrete direction specified by $\alpha$. In this study, all simulations are carried out in two-dimensional system. Accordingly, we employed the two-dimensional nine-velocity (D2Q9) model in which the discrete velocity vectors are defined on the two-dimensional square lattice space as $\boldsymbol{e}_{0}=(0,0), \boldsymbol{e}_{\alpha}=(\cos [(\alpha-1) \pi / 2], \sin [(\alpha-1) \pi / 2]) c$ with $\alpha=1-4$ and $\boldsymbol{e}_{\alpha}=(\cos [(\alpha-5) \pi / 2+\pi / 4], \sin [(\alpha-5) \pi / 2+\pi / 4]) c$ with $\alpha=5-8$. Here, $c$ is given as $c=\delta x / \delta t$ with the lattice spacing $\delta x$. In Eq. (5), $\mathbf{x}$ is the lattice coordinate, $\delta t$ is the time step and $\tau$ is the relaxation time which is given as

$$
\tau=0.5+3 v \frac{\delta t}{\delta x^{2}}
$$

where $v$ is the kinematic viscosity. Also, $f_{\alpha}^{(e q)}$ is the equilibrium distribution function given as [11] 


$$
f_{\alpha}^{(e q)}=\omega_{\alpha}\left\{\rho+\rho_{0}\left[\frac{\mathbf{e}_{\alpha} \cdot \mathbf{u}}{c_{s}{ }^{2}}+\frac{1}{2} \frac{\left(\mathbf{e}_{\alpha} \cdot \mathbf{u}\right)^{2}}{c_{s}{ }^{4}}-\frac{1}{2} \frac{\mathbf{u} \cdot \mathbf{u}}{c_{s}{ }^{2}}\right]\right\}
$$

where $\omega_{\alpha}$ is the weight function given as $\omega_{0}=4 / / 9, \omega_{\alpha}=1 / 9$ with $\alpha=1-4$ and $\omega_{\alpha}$ $=1 / 36$ with $\alpha=5-8$ and $c_{s}$ is given by $c_{s}{ }^{2}=c^{2} / 3 . F_{\alpha}$ is the force term of $\alpha$ dependent, which is given by

$$
F_{\alpha}=\omega_{\alpha} \rho_{0}\left(1-\frac{1}{2 \tau}\right)\left[\frac{\mathbf{e}_{\alpha} \cdot \mathbf{F}}{c_{s}^{2}}+\frac{\mathbf{u F}:\left(\mathbf{e}_{\alpha} \mathbf{e}_{\alpha}-c_{s}^{2} \mathbf{I}\right)}{\left(1-f_{s}\right) c_{s}{ }^{2}}\right]
$$

where the symbol, : , represents Frobenius inner product, $\mathbf{I}$ is the identity matrix and $\mathbf{F}$ is the applied force as given by

$$
\mathbf{F}=\left(1-f_{s}\right)\left(-\frac{v}{K} \mathbf{u}+\frac{\rho_{0}+\delta \rho}{\rho_{0}} \mathbf{g}\right)
$$

The density $\rho$ is calculated by $\rho=\Sigma_{\alpha} f_{\alpha}$ and the velocity vector $\boldsymbol{u}$ can be calculated as follows,

$$
\rho_{0} \mathbf{u}=\left(\sum_{\alpha} \mathbf{e}_{\alpha} f_{\alpha}+\frac{\delta t}{2}\left(1-f_{s}\right)\left(\rho_{0}+\delta \rho\right) \mathbf{g}\right) /\left(1+\left(1-f_{s}\right) \frac{\delta t}{2} \frac{v}{K}\right)
$$

This is the LBM-coupled model developed in the previous work [15]. This model is consistent with Eqs. (1) and (2) with $\beta=0$, i.e., with no solidification shrinkage.

In this study, we construct new LBM that recovers Eqs. (1) and (2) with $\beta \neq 0$. For this, the Chapman-Enskog analysis [14] was carried out by expanding $f_{\alpha}$, time and spatial scales as $f_{\alpha}=f_{\alpha}{ }^{0}+\varepsilon f_{\alpha}{ }^{(1)}+\varepsilon^{2} f_{\alpha}{ }^{(2)}+\cdots, \partial / \partial t=\varepsilon \partial / \partial t_{1}+\varepsilon^{2} \partial / \partial t_{2}$ and $\partial / \partial x=\varepsilon \partial / \partial x_{1}$ with $\varepsilon$. Here, $\varepsilon$ is the expansion parameter proportional to the ratio of the lattice spacing to a characteristic macroscopic length. The analysis of Eq. (5) for each order of $\varepsilon$ yields macroscopic equations, from which one can modify and/or introduce terms necessary to recover the desirable macroscopic relations. The analysis carried out in this study is essentially the same as the standard one and some details are explained in Appendix A. In this study, the effect of solidification shrinkage is introduced by replacing Eq. (8) with the following equation, 


$$
\begin{aligned}
F_{\alpha}= & \omega_{\alpha}\left(1-\frac{1}{2 \tau}\right)\left[\frac{\mathbf{F} \cdot \mathbf{e}_{\alpha}}{c_{s}{ }^{2}}+\frac{\mathbf{u F}:\left(\mathbf{e}_{\alpha} \mathbf{e}_{\alpha}-c_{s}{ }^{2} \mathbf{I}\right)}{\left(1-f_{s}\right) c_{s}{ }^{4}}\right] \\
& +\omega_{\alpha} \beta \frac{\partial f_{s}}{\partial t}\left[\frac{1}{2} \frac{\mathbf{I}:\left(\mathbf{e}_{\alpha} \mathbf{e}_{\alpha}-c_{s}{ }^{2} \mathbf{I}\right)}{c_{s}{ }^{2}}-1\right]
\end{aligned}
$$

The Chapman-Eskog analysis shows that the model with Eq. (11) can recover the macroscopic NS Eq. (2) and the following continuity equation,

$$
\frac{\partial \rho}{\partial t}+\nabla \cdot(\rho \mathbf{u})=-\beta \frac{\partial f_{s}}{\partial t}+\Delta E_{c}
$$

where

$$
\Delta E_{c}=\frac{1}{2} \varepsilon^{2} \delta t \beta \frac{\partial}{\partial t_{1}}\left(\frac{\partial f_{s}}{\partial t_{1}}\right)
$$

Here, the term $\varepsilon^{2}$ and $\delta$ are small parameters and, therefore, the term proportional to $\varepsilon^{2} \delta$, i.e., $\Delta E_{c}$ is negligible as usual [23]. Therefore, the continuity Eq. (1) with $\beta \neq 0$ is recovered in this model.

As describe above, LBM with Eqs. (5)-(7) and Eqs. (9)-(11) formally recovers the macroscopic Eqs. (1) and (2). However, this model is not free from the problem with the numerical stability. One of factors that affect the numerical stability of LBM is the value of $\tau$ given by Eq. (6) which must be larger than 0.5 . The numerical simulation of LBM is unstable when $\tau$ is very close to 0.5 and, hence, $\delta x$ and $\delta t$ must be chosen in terms of the numerical stability for a given value of $v$. On the other hand, $\delta x$ and $\delta t$ need to be determined in light of the numerical stability of Eq. (3) (and also Eq. (4)). More specifically, the numerical simulation of Eq. (3) based on a simple Euler method, which is suitable for parallel computing, requires $\delta t \leq \xi \delta x^{2} / a_{T}$ with diffusion number $\xi$. It results in the requirement $\operatorname{Pr} \leq(\tau-0.5) /(3 \xi)$ where $\operatorname{Pr}=v / a_{T}$ is the Prandtl number [15]. For example, when 0.65 is assigned to $\tau$, Pr must be $0.1-0.3$ which are about one order of magnitude larger than typical values in metallic systems. Hence, in our previous simulation, a fluid with high viscosity was simulated to avoid this problem.

A number of efforts have been devoted to improving the numerical stability of LBM. For instance, the numerical simulation of LBM can be stabilized by employing multiple relaxations times in the so-called Multiple-Relaxation-Time(MRT)-LBM [24]. In terms of computational efficiency, in this study, we focus on a Modified Lattice Bhadnager Gross Krook (MLBGK) method [16] in which a single relaxation time is employed, while a new correction parameter is introduced to improve the numerical 
stability. Hence, we construct the macrosegregation simulation model based on the MLBGK method. This is achieved by replacing Eqs. (6) and (7) with the following equations [16], respectively,

$$
\begin{aligned}
& \tau=0.5+3 v \frac{\delta t}{\delta x^{2}}+\gamma \\
& f_{\alpha}^{(e q)}= \begin{cases}\rho_{0}-\left(1-\omega_{\alpha}\right)\left(1-f_{s}\right) p / c_{s}{ }^{2}+\rho_{0} s_{0}(\mathbf{u})+\rho_{0} r_{0}(\mathbf{u}) & \alpha=0 \\
\omega_{\alpha}\left(1-f_{s}\right) p / c_{s}{ }^{2}+\rho_{0} s_{\alpha}(\mathbf{u})+\rho_{0} r_{\alpha}(\mathbf{u}) & \alpha \neq 0\end{cases}
\end{aligned}
$$

where $\gamma$ is the newly introduced correction parameter to improve the numerical stability. $s_{\alpha}(\mathbf{u})$ and $r_{\alpha}(\mathbf{u})$ are given by

$$
\begin{aligned}
& s_{\alpha}(\mathbf{u})=\omega_{\alpha}\left(\frac{\mathbf{e}_{\alpha} \cdot \mathbf{u}}{c_{s}^{2}}+\frac{1}{2} \frac{\mathbf{u u}:\left(\mathbf{e}_{\alpha} \mathbf{e}_{\alpha}-c_{s}{ }^{2} \mathbf{I}\right)}{\left(1-f_{s}\right) c_{s}{ }^{4}}\right) \\
& r_{\alpha}(\mathbf{u})=\gamma \delta t \omega_{\alpha} \frac{\mathbf{S}:\left(\mathbf{e}_{\alpha} \mathbf{e}_{\alpha}-c_{s}{ }^{2} \mathbf{I}\right)}{2 c_{s}{ }^{2}}
\end{aligned}
$$

with the shear rate $\mathbf{S}$ defined as

$$
\mathbf{S}=\frac{\sum_{\alpha} \mathbf{e}_{\alpha} \mathbf{e}_{\alpha}\left(f_{\alpha}-f_{\alpha}^{e(0)}\right)+\frac{1}{2} \delta t \rho_{0}(\mathbf{u F}+\mathbf{F u}) /\left(1-f_{s}\right)}{c_{s}^{2} \rho_{0}(\gamma-\tau) \delta t}
$$

where $f_{\alpha}{ }^{e(0)}$ is given by Eq. (15) with $r_{\alpha}=0$. The pressure $p$ in Eq. (15) is calculated as follows,

$$
p=\frac{c_{s}^{2}}{\left(1-f_{s}\right)\left(1-\omega_{0}\right)}\left(\sum_{\alpha \neq 0} f_{\alpha}+\tau \delta t F_{0}+\rho_{0} s_{0}(\mathbf{u})\right)
$$

In summary, the present LBM-coupled model consists of Eqs. (5), (9)-(11) and (14)-(19). Since the MLBGK model exhibits better numerical stability for simulations of fluid dynamics than the standard LBM [16], the numerical stability of macrosegregation simulation using the present model should be better than that using the previous LBM-coupled model. In the present model, $\gamma$ in Eq. (14) (also in Eqs. (17) and (18)) is the important parameter affecting the numerical stability. It is understood from Eq. (17) that $\gamma$ determines the contribution of shear rate to the equilibrium distribution function. It was shown in the previous work [16] that the numerical stability can be improved when $\gamma$ is a finite but close to 0 . We carried out simulations with different values of $\gamma$ for the cases of lateral directional solidification and continuous casting to see its effect on the stability and accuracy of the simulations. The boundary condition in the simulations 
of the present model was calculated based on the nonequilibrium extrapolation scheme [16].

The necessary steps to calculate this model is summarized as follows,

1.The computational parameters and domain of the computation are initialized.

2. The time derivative of solid fraction, $\partial f_{s} / \partial t$, is calculated [15].

3. The energy conservation and solute conservation equations (3) and (4) are solved.

4. The fluid velocity is calculated based on the LBM with the boundary condition.

5. The temperature $T$, solute concentration $C_{L}$ and the distribution function $f_{\alpha}$ are updated.

6. Steps 2 to 5 are repeated until the end of the simulation.

Note that our model is based on the D2Q9 model and is restricted to two-dimensional simulations. Although some problems can be well analyzed by two-dimensional simulations, three-dimensional simulations are required for analyses of most of problems. In this regard, it is necessary to apply the present model to three-dimensional system, for instance, based on D3Q19 model. This remains as an important future work. In addition, as described above, $\gamma$ is the important parameter affecting the numerical stability of the model. Although it was found in the previous work [16] that the value of $\gamma$ close to 0 yields reasonable accuracy, an appropriate value of $\gamma$ must be found for each of problem by carrying out preliminary simulations when this model is applied to new problems.

\section{Steady state flow}

We investigate the accuracy and the numerical stability of the present LBM-coupled model, focusing on the steady-state flow between parallel planes in fluid systems and in a solid and liquid two-phase system. The computational system consists of 128 and 16 lattice points in $x$ - and $y$-directions, respectively, which is sandwiched between two planes parallel in $x$-direction. A non-slip boundary condition was applied to both planes (at $y=0$ and $y=16 \delta x$ ). For the sake of convenience, all quantities are normalized by $c_{s}$, $\delta t$ and $\rho$. Although we calculated the steady-state flow for different kinematic viscosity and pressure difference, we show only representative results that are sufficient for discussion about the numerical accuracy of the present model. The dimensionless kinematic viscosity $v^{*}=v /\left(c_{s}^{2} \delta t\right)$ was set to $v^{*}=0.15$ and the dimensionless pressure $p^{*}=p /\left(\rho c_{s}{ }^{2}\right)$ at $x=0$ and $128 \delta x$ was set to 0.11 and 0.10 , respectively. The correction parameter $\gamma$ was set to $\gamma=0.01$. The simulation was carried out until the steady-state of $\mathbf{u}$ field was realized. The results shown in Fig. 1(a) are the spatial profiles of $u_{x}$ in $y$-direction at $x=64 \delta x$ in the purely fluid system obtained from 
the present model (full circles) and the NS-based model (squares). The well-known analytical solution of this Poiseuille flow is indicated by the solid line. The result of the present model is in good agreement with those of the NS-based model and the analytical model. It supports the validity of the present model for calculation of the viscous fluid.

In Fig. 1(a), open circles represent the result of the previous LBM-coupled model [15] which is consistent with those of the NS-based model and the analytical solution. As described in Sec. 2, the problem with the previous LBM-coupled model is low numerical stability. The previous model cannot be applied to flows at high Reynolds number in a fluid with low kinematic viscosity as is in the case of the standard LBM. Accordingly, this model cannot be applied to simulations of metallic alloy systems. When the Reynolds number $R e$ is defined as $R e=L u$ ' $/ v$ with the distance between two planes $L$ and $u^{\prime}$ is a half of the maximum value of $u_{x}$ at $x=64 \delta x$, Re in Fig. 1(a) corresponds to $R e=4.6$. We carried out the simulation of Poiseuille flow with lower kinematic viscosity than that employed in Fig. 1(a). To be more specific, $v^{*}$ was set to $v^{*}=0.03$. The results are shown in Fig. 1(b). In this condition, $R e$ is given as $R e=$ 115.5 and the solution of the previous LBM-coupled model cannot be obtained because it is numerically not stable. However, the present model is stable even in this condition and its result is in good agreement with the results of analytical solution and NS-based model as shown in Fig. 1(b). It demonstrates that that the numerical stability of the present LBM-coupled model is better than that of the previous model.

Next, we focus on the steady-state flow in a solid and liquid two-phase system where the Darcy's term arises. The computational system and conditions are the same as those used in the calculation of Fig. 1(a), except that the volume fraction was set to 0.5. The dimensionless permeability $K^{*}=K /\left(c_{s} \delta t\right)^{2}$ was given as $K^{*}=0.0225$. The results are shown in Fig. 2 where the following analytical solution for the steady state flow [17] is indicated by the solid line.

$$
u_{x}=\frac{1}{\rho} \frac{d p}{d x} \frac{K}{v}\left(1-e^{-\sqrt{y / K}}\right)\left(e^{-\sqrt{y / K}}-e^{-\sqrt{L_{y} / K}}\right) /\left(1-e^{\sqrt{L_{y} / K}}\right)
$$

where $L_{y}=16 \delta x$. The result of the present model is in good agreement with those of the NS-based model and the analytical model. Hence, the accuracy of the present LBM-coupled model is sufficiently high. In Fig. 2, the result of the previous model oscillates near the wall, indicating that it is numerically not stable in this condition. Therefore, the numerical stability of the present LBM-coupled model is improved as compared with that of the previous model in the case of solid and liquid two-phase systems.

Although not shown here because of limitation of space, the steady-state flow 
under the temperature gradient, i.e., thermal convection was studied as in the previous work [15]. The result of the present model is in good agreement with the one of NS-based model.

Note that the data of NS-based model are shifted by $1 / 2 \delta x$ in Figs. 1 and 2. This is because the staggered grid was employed to solve the NS equation, while the regular grid was employed in LBM. This difference in the type of grid does not have any influence on the results of steady-state flows as shown in Figs. 1 and 2. However, it causes differences of non-steady-state flow between both methods especially near the boundaries (wall) as shown in the next section.

\section{Lateral directional solidification of a Sn-Bi alloy}

\subsection{Computational details}

Formation of channel segregation during lateral directional solidification of Sn-Bi alloy was studied by means of experiments and numerical simulations in Ref. [18] where the NS-based model explained in Sec. 2.1 was employed. Note that the previous LBM-coupled model cannot be applied to this simulation because of the low numerical stability. It is important to test to see whether or not the present LBM-coupled model can describe the formation behavior of the channel segregation in this system. It is also important to clarify how much acceleration can be achieved in the simulation of this realistic process. Therefore, we carried out the simulations for this process, using the present LBM-coupled model and the NS-based model.

In this study, we focus on Sn-10mass\%Bi alloy. The liquidus temperature was given as a function of $\mathrm{Bi}$ composition and also the partition coefficient was given as a function of temperature as described in in Ref. [18]. In this calculation, $\beta$ in Eqs. (1) and (11) was set to $\beta=0$ (no solidification shrinkage) and $\delta \rho$ in Eqs. (2) and (9) were given as $\delta \rho=\left\{\rho_{\mathrm{Sn}} C_{L}+\rho_{\mathrm{Bi}}\left(1-C_{L}\right)\right\}\left\{1-\beta_{T}\left(T-T_{R e f}\right)\right\}$ where $\rho_{\mathrm{Sn}}$ and $\rho_{\mathrm{Bi}}$ are densities of Sn and $\mathrm{Bi}$ atoms, respectively, $\beta_{T}$ is the thermal expansion coefficient and $T_{R e f}$ is the reference temperature. All parameters employed in this study are listed in Table 1 . We used a two-dimensional system of $64 \times 64 \mathrm{~mm}^{2}$. The non-slip boundary condition was applied to all boundaries. The adiabatic condition was applied to all boundaries except for the left-side boundary at which the temperature was decreased at a constant rate of $5 \mathrm{~K} / \mathrm{s}$. The initial velocity and the initial temperature were uniformly set to $0 \mathrm{~m} / \mathrm{s}$ and $523 \mathrm{~K}$, respectively, over the whole system. All simulations were stopped at $t=1600 \mathrm{~s}$ because the solidification finished by $1600 \mathrm{~s}$ in all simulations.

We performed the simulations for different grid spacing $\delta x$ and time step $\delta t$ to evaluate the computational speed of the NS-based and the present LBM-coupled models. 
In this study, $\delta x$ and $\delta t$ were chosen so that the simulation of the NS-based model finishes in a reasonable computation time because it took much longer computational time than the present model. The conditions are listed in Table 2 where values of $\tau$ are also described. In order to examine acceleration by parallel computing, both serial computing on CPU and parallel computing on Graphics Processing Unit (GPU) were conducted in all cases. However, it was not possible to obtain the result of case 3 by the NS-based model because it requires extremely long computational time. As described in Sec. 2.1, Eqs. (1) and (2) were solved by the SIMPLE method in the NS-based model, where the Jacobi iterative method was employed for the GPU computation. We employed GPU Tesla P100 for all GPU computations. In addition, we investigated an effect of $\gamma$ on the result of the LBM-coupled model, focusing on the range of $\gamma$ from 0 to 1.0 .

\subsection{Simulation results}

The result of the present LBM-coupled model is shown in Fig. 3. This is the result of GPU computation. Figures 3(a), (b) and (c) represent the change of solid fraction (gray scale) and the velocity $\mathbf{u}$ (arrow) during the solidification, while Figs. 3(d), (e) and (f) show the change of segregation ratio $C_{\text {seg }}$ defined as local volume averaged molar composition of Bi divided by the average composition of the alloy. The formation of channel segregation is observed in Fig. 3. Let us stress that it is not possible to simulate this process using the previous LBM-coupled model because of the low numerical stability. We found that the numerical simulation of the present LBM-coupled model is stable in the range of $\gamma$ between 0.05 and 0.1 and the result does not substantially depend on $\gamma$ in this range. Hence, the result only for $\gamma=0.05$ is shown in Fig. 3.

The solidification starts from the left-side wall and it proceeds rightward in Fig. 3. As the solidification proceeds, $\mathrm{Bi}$ atom is rejected from the solid into the liquid. Accordingly, the liquid with high Bi composition, which has a high density, flows downward. Then, it leads to an anti-clockwise flow ahead of the solidification front. Importantly, local non-uniformity of composition emerges due to such convection of liquid. Once relatively high composition region forms, the liquidus temperature become low in this region, thus causing the delay of solidification, i.e., low solid fraction. Bi composition in this region further increases due to solute rejection accompanied by the solidification in the vicinity of this region. Hence, the liquidus temperature further decreases in this region. This is the mechanism for the formation of channel segregation observed in Fig. 3. It is also important to notice that relatively high composition region 
forms in the lower right part of ingot. This positive segregation is caused by the rightward flow of high composition liquid oozed from the channel along the bottom edges.

The segregation patterns at $1600 \mathrm{~s}$ calculated by the NS-based model and LBM-based model are compared in Fig. 4. These results were obtained from the GPU computations. The reasonable agreement between them is observed. More specifically, in both cases, the channel segregation emerges in the lower left part, and also, the relatively high composition region (positive segregation) forms in the lower right part. On the other hand, from a closer look at both results, one can see the quantitative difference between them. The composition in the lower right part is low, and accordingly, the composition in the channel segregation is high in the result of LBM-coupled model as compared with the one of NS-based model. Although not shown here, the area of positive segregation region in the lower right part is slightly reduced with increasing the number of grid points from $64^{2}$ to $128^{2}$ in the NS-based model. In the LBM-coupled model, on the other hand, this positive segregation region is extended and the composition in this region gradually increases as the number of grid points increases from $64^{2}$ to $256^{2}$. Namely, the quantitative difference between them gradually decreases as the number of grid points increases. Hence, the quantitative difference between the LBM-coupled model and NS-based model observed in Fig. 4 is ascribable to numerical errors in both models originating from coarse grid spacing. Note that the positive segregation in the lower right part of ingot originates from the rightward flow of high composition liquid from the channel along the bottom edges. The flow near the edge is sensitive to the type of grid (staggered or regular). When the coarse grid is employed, therefore, the difference in the flow near the bottom edge appears, causing the difference in the segregation in the lower right part and thus the final composition in the channel segregation. It is expected that the results of the LBM-coupled model and NS-based model should coincide with each other when sufficiently fine grid spacing is employed. However, we did not carry out the simulations with the finer grid spacing because the long computational time is required especially in the NS-based model.

The main purpose of this study is the acceleration of macrosegregation simulations. The computational times are plotted in Fig. 5 where $x$ axis is the total number of grid points multiplied by the total number of time steps that is a measure of the computational cost. For reference, the number of grid points $N_{\text {grid }}$ is denoted at the top of the figure. Circles and squares are the results of NS-based and LBM-coupled models, respectively. Also, the CPU and GPU computations are represented by the open 
and full symbols, respectively. We employed CPU Xeon E5-1620 V3 $3.50 \mathrm{GHz}$ and GPU Tesla K20 in CPU and GPU computations, respectively. One can understand that the significant acceleration is achieved in the present LBM-coupled model. In both models, the computational time is reduced by performing parallel computation on GPU. However, when the simulation with $128^{2}$ gird points is considered, the computational time of NS-based model on GPU is longer than the one of the LBM-coupled model even on CPU. Moreover, when the GPU computation with $128^{2}$ grid points is considered, the LBM-coupled model is about 34 times faster than the NS-based model. Therefore, the acceleration is successfully achieved in simulations for this realistic process.

\section{Continuous casting}

\subsection{Computational details}

The macrosegregation in continuously cast slab of $\mathrm{Fe}-0.55$ mass\% $\%$ steel was recently investigated by the numerical simulation using the NS-based model explained in Sec. 2.1 [19]. Flows induced by thermo-solutal convection and solidification shrinkage were taken into account in the recent work. In this study, we performed the same simulation by means of the present LBM-coupled model and compare the computational time with the one of the NS-based model reported in Ref. [19].

The computational condition employed in this study is basically the same as that in the recent work [19]. As illustrated in Fig. 6(a), the computational system consists of two-dimensional box with a $2 \mathrm{~m}$ length in $x$-direction and $0.25 \mathrm{~m}$ thickness in $y$-direction. The right-side wall is a chill wall, while the left-side, the upper and lower walls are adiabatic walls. The cooling process in the continuous casting was approximated by moving the chill zone on the upper and lower walls from right to left. The moving velocity of the chill zone corresponds to the casting speed which was set to $1 \mathrm{~m} / \mathrm{min}$. The inlet of melt was considered on the left-side wall to describe the incoming flow that compensates for the volume reduction due to solidification shrinkage. The size of inlet was set to $5 \delta x$ and it feeds the melt having the initial composition. Note that the velocity of the melt coming out of the inlet is determined by the solidification shrinkage. The continuous caster is schematically illustrated in Fig. 6(b), the spatial coordinate of which is rotated at $90^{\circ}$ from the one in Fig. 6(a). Since it is a curved-type continuous caster, the gravity direction changes with time from $x$-direction to $-y$-direction in the computational box of Fig. 6(a). The total time of casting process was set to $1500 \mathrm{~s}$. All parameters employed in the simulation are shown in Table 3. The simulations were accelerated by parallel computing on Tesla P100 GPU. 
In the previous work [19], the simulation of NS-based model was carried out with the grid spacing of $\delta x=5$ and $10 \mathrm{~mm}$. It was found that the simulation with $\delta x=$ $10 \mathrm{~mm}$ is numerically unstable. Therefore, only the simulation result with $\delta x=5 \mathrm{~mm}$ was discussed in the previous work. As pointed out by the authors, however, the checkerboard patterns of segregation ratio, which stems from the numerical error, were observed even in the simulation with $\delta x=5 \mathrm{~mm}$. Therefore, the grid spacing $\delta x=5 \mathrm{~mm}$ is not fine enough to obtain the accurate result for this process. In fact, our preliminary simulation showed that the simulation with $\delta x=5 \mathrm{~mm}$ is unstable in the present LBM-coupled model. Accordingly, the result shown in this paper is the one obtained with $\delta x=2.5 \mathrm{~mm}$ and $\delta t$ was set to $0.5 \mathrm{~ms}$. We investigated effect of $\gamma$ and found that the simulation is stable in the range between $\gamma=0.005$ and 0.05 and the result does not significantly depend on the value of $\gamma$ in this range. Therefore, only the result obtained for $\gamma=0.01$ will be discussed below.

\subsection{Numerical results}

Figure 7 represents the simulation result of the present LBM-coupled model. The distributions of $f_{s}$, the magnitude of fluid velocity $|\mathbf{u}|$ and $C_{\text {seg }}$ of $\mathrm{C}$ at three time steps are shown. The solidification gradually takes place from the upper and lower walls into the center part of $y$-direction. In other words, the solidification shell becomes thick with time. At 800 and $1200 \mathrm{~s}$, the shape of growth front of shell is not even but fine wedge shape. The thermo-solutal convection causes the spiral-shaped flow as can be understood from $|\mathbf{u}|$ patterns. This spiral-shaped flow yields V-shaped non-uniformity of C composition. This formation mechanism of the V-shaped non-uniformity of $\mathrm{C}$ composition is essentially identical to that of channel segregation shown in Fig. 3. Also, the positive segregation region of $\mathrm{C}$ forms along the centerline of the slab at $1200 \mathrm{~s}$. These behaviors are comparable with those described by the NS-based model [19]. The segregation pattern at $1200 \mathrm{~s}$ (Fig. 7(c)) is very similar to the result of NS-based model (Fig. 8(a) of Ref. [19]). However, there are quantitative differences. The maximum (minimum) composition in the positive (negative) segregation in the present model is higher (lower) than the one of the NS-based model. Note that the accuracy of the previous simulation of NS-based model is not sufficiently high as pointed out by the authors. Also, the difference in the type of spatial grid (staggered or regular) for computation of fluid flow causes the difference in fluid dynamics when the gird is not sufficiently fine as described in Sec. 4. A further discussion on this point requires computation of NS-based model with fine grid spacing which is extremely time-consuming as will be understood below. It remains as an important future work. It 
should be stressed that both models consistently predict the formations of V-shaped and centerline segregation in this continuous casting process.

Let us discuss the computational time. It was reported that the simulation of this process until $t=1160 \mathrm{~s}$ took 1497 hours by the NS-based model, even though the simulation was accelerated by a shared-memory parallel computing with 6 threads (Xeon $54723.0 \mathrm{GHz}$ ). On the other hand, the simulation of the present LBM-coupled model took only 1.5 hours on GPU. Surprisingly, therefore, the simulation by the present model is about 1,000 times faster than the one by the NS-based model. Although a part of this remarkable speedup might be ascribed to a difference in performance of the computer, it is evident that the extremely fast computation was achieved by the present LBM-coupled model.

It was demonstrated that the present LBM-coupled model allows for fast computations for realistic problems of casting. This model can be applied to the simulations for very large system with fine grid spacing that has been not been possible by means of the NS-based models.

\section{Conclusions}

To realize fast computation of macrosegregation simulation, we developed a numerical model by coupling solute and energy conservation equations with Lattice-Boltzmann Method (LBM). The previous LBM-coupled model [15] cannot be applied to metallic alloy systems because of low numerical stability. This problem was resolved in this study to employ modified lattice Bhadnager-Gross-Krook method [16]. Also, the solidification shrinkage was taken into account. The model was validated by comparing the results for steady-state flows with those of analytical solutions and a model based on the Navier-Stokes (NS) equation. The simulations for lateral directional solidification of Sn-Bi alloy were carried out. The present model successfully describes the formation of channel segregation as is consistent with the NS-based model. The computational times of the present model were much shorter than those of the NS-based model. When the GPU computation with $128^{2}$ grid points is considered, the LBM-coupled model is about 34 times faster than the NS-based model. In this study, also, we conducted the simulations for continuous casting of a steel slab, where V-shaped segregation and centerline segregation were reproduced. In this case, the simulation by the present model is about 1,000 times faster than the one by the NS-based model. Hence, the remarkable acceleration was achieved. It is expected that the present model is applied to a variety of macrosegregation problems in metallic alloy systems. 
Before closing, it should worthwhile pointing out the following issues. In lateral solidification of Sn-Bi alloy and continuous casting of a steel slab, reasonable agreements were found between the segregation distributions predicated by the present LBM-coupled model and the NS-based model. However, there are quantitative disagreements between them. In the case of lateral solidification, the differences gradually diminish as the grid spacing decreases. Hence, the quantitative differences should stem from the numerical errors in both models associated with coarse grid spacing. A further investigation should be aimed at elimination of the quantitative differences. Next, in this study (also in the previous study), we used LBM for fluid dynamics in porous media in Ref. [14] in order to include effect of solid fraction. Although our LBM-coupled model is consistent with the NS-based model reported in Refs. [17-19], it is important to develop the LBM-coupled model equivalent to the other NS-based models that include effects of solid fraction in different ways. In this regard, the full volume-averaged Navier-Stokes equation can be reproduced by LBM developed in Ref. [25]. Hence, the construction of the LBM-coupled model based on such LBM should be important task to be tacked in a future work. In addition, LBM is suitable for parallel computing and, accordingly, our simulations were accelerated by GPU computation in this study. Recently, LBM was coupled with a phase-field method to simulate solidification microstructures under fluid flows [26, 27] and the simulations were conducted using massively parallel GPU (i.e., multi GPU) computing technique [28]. Coupled with multi-GPU computation technique, the present model will contribute to further progress in our understanding of the formation processes of macrosegregation.

\section{Acknowledgements}

This research was supported by $25^{\text {th }}$ ISIJ research promotion grant. 


\section{Appendix A. Chapman-Enskog analysis}

In this appendix, we derive Eq. (11) by carrying out Chapman-Enskog expansion of Eq. (5). In D2Q9 model, the following relations are satisfied,

$$
\begin{aligned}
& \sum_{\alpha=0}^{8} \omega_{\alpha} e_{\alpha, i}=0 \\
& \sum_{\alpha=0}^{8} \omega_{\alpha} e_{\alpha, i} e_{\alpha, j}=c_{s}{ }^{2} \delta_{i j} \\
& \sum_{\alpha=0}^{8} \omega_{\alpha} e_{\alpha, i} e_{\alpha, j} e_{\alpha, k}=0 \\
& \sum_{\alpha=0}^{8} \omega_{\alpha} e_{\alpha, i} e_{\alpha, j} e_{\alpha, k} e_{\alpha, l}=c_{s}{ }^{4}\left(\delta_{i j} \delta_{k l}+\delta_{i k} \delta_{j l}+\delta_{i l} \delta_{j k}\right)
\end{aligned}
$$

Therefore, the equilibrium distribution function given by Eq. (7) satisfies the following relations,

$$
\begin{aligned}
& \sum_{\alpha} f_{\alpha}^{e q}=\rho \\
& \sum_{\alpha} e_{\alpha, i} f_{\alpha}^{e q}=\rho_{0} u_{i} \\
& \sum_{\alpha} e_{\alpha, i} e_{\alpha, j} f_{\alpha}^{e q}=\rho c_{s}^{2} \delta_{i j}+\frac{1}{f_{l}} \rho_{0} u_{i} u_{j} \\
& \sum_{\alpha} e_{\alpha, i} e_{\alpha, j} e_{\alpha, k} f_{\alpha}^{e q}=c_{s}^{2} \rho_{0}\left(\delta_{i j} u_{k}+\delta_{i k} u_{j}+\delta_{j k} u_{i}\right)
\end{aligned}
$$

To obtain the macroscopic equations (1) and (2), we first consider the following form of the force term [29],

$$
F_{\alpha}=\omega_{\alpha}\left[A+\frac{\mathbf{B} \cdot \mathbf{e}_{\alpha}}{c_{s}{ }^{2}}+\frac{1}{2} \frac{\mathbf{C}:\left(\mathbf{e}_{\alpha} \mathbf{e}_{\alpha}-c_{s}{ }^{2} \mathbf{I}\right)}{f_{l} c_{s}{ }^{4}}\right]
$$

where a scalar $A$, a vector $\mathbf{B}$ and a matrix $\mathbf{C}$ will be determined later so as to derive the macroscopic equations of interest. Also, the fluid velocity is defined as

$$
\rho_{0} \mathbf{u}=\sum_{\alpha} \mathbf{e}_{\alpha} f_{\alpha}+m \delta t \mathbf{F} \quad \text { (A. 10) }
$$

where $m$ is a constant that will be determined later.

In order to obtain the macroscopic equations from Eq. (5), the distribution function $f_{\alpha}$, time scale $t$ and spatial scale $\mathbf{x}$ are expanded using the expansion parameter $\varepsilon$ as follows, 


$$
\begin{aligned}
& f_{\alpha} \approx f_{\alpha}^{(0)}+\varepsilon f_{\alpha}^{(1)}+\varepsilon^{2} f_{\alpha}^{(2)}+\cdots \\
& \frac{\partial}{\partial t}=\varepsilon \frac{\partial}{\partial t_{1}}+\varepsilon^{2} \frac{\partial}{\partial t_{2}} \\
& \frac{\partial}{\partial \mathbf{x}}=\varepsilon \frac{\partial}{\partial \mathbf{x}_{1}}
\end{aligned}
$$

In addition, $A, \mathbf{B}$ and $\mathbf{C}$ in Eq. (A. 9) are explained by $\varepsilon$ as $A \approx \varepsilon A_{1}+\varepsilon^{2} A_{2}, \mathbf{B} \approx \varepsilon \mathbf{B}_{1}$ and $\mathbf{C} \approx \varepsilon \mathbf{C}_{1}+\varepsilon^{2} \mathbf{C}_{2}$. Here, as will be realized later, $A$ and $\mathbf{C}$ are related to $\partial f_{s} / \partial t$ and hence the expansions were carried out up to the second order. Accordingly, the force term is expanded as $F_{\alpha} \approx \varepsilon F_{\alpha, 1}+\varepsilon^{2} F_{\alpha, 2}$. In addition, the applied force $\mathbf{F}$ is given as $\mathbf{F} \approx \varepsilon \mathbf{F}_{1}$.

By expanding $f_{\alpha}$ in Eq. (5) about $\mathbf{x}$ and $t$ and by substituting above expansions into the resulting equation, one can obtain the following equation in each order of $\varepsilon$,

$$
\begin{array}{ll}
O\left(\varepsilon^{0}\right): & f_{\alpha}^{(0)}=f_{\alpha}^{(e q)} \quad(\text { A. 14) } \\
O\left(\varepsilon^{1}\right): & D_{\alpha} f_{\alpha}^{(0)}=-\frac{f_{\alpha}^{(1)}}{\tau \delta t}+F_{\alpha, 1} \quad \text { (A. 15) } \\
O\left(\varepsilon^{2}\right): & \frac{\partial f_{\alpha}^{(0)}}{\partial t_{2}}+\left(1-\frac{1}{2 \tau}\right) D_{\alpha} f_{\alpha}^{(1)}+\frac{1}{2} \delta t D_{\alpha} F_{\alpha, 1}=-\frac{f_{\alpha}^{(2)}}{\tau \delta t}+F_{\alpha, 2}
\end{array}
$$

where

$$
D_{\alpha}=\frac{\partial}{\partial t_{1}}+\mathbf{e}_{\alpha} \cdot \frac{\partial}{\partial \mathbf{x}_{1}}
$$

From the definition of $\rho$, Eqs. (A.5), (A. 10) and (A.14), one finds,

$$
\begin{aligned}
& \sum_{\alpha} f_{\alpha}^{(k)}=0, \quad \sum_{\alpha} \mathbf{e}_{\alpha} f_{\alpha}^{(k)}=0 \quad \text { for } \quad k \geq 1 \quad \text { (A. 18) } \\
& \sum_{\alpha} f_{\alpha}^{(1)} \mathbf{e}_{\alpha}=-m \delta t \mathbf{F}_{1} \quad \text { (А.19) }
\end{aligned}
$$

By taking moments of Eq. (A.15), then, one can obtain the macroscopic equations

$$
\begin{aligned}
& \frac{\partial \rho}{\partial t_{1}}+\nabla\left(\rho_{0} \mathbf{u}\right)=A_{1} \quad \text { (A. 20) } \\
& \frac{\partial\left(\rho_{o} \mathbf{u}\right)}{\partial t_{1}}+\nabla \cdot \Pi_{0}=\left(\frac{m}{\tau}+n\right) \mathbf{F}_{1}
\end{aligned}
$$

where $\Pi_{0}$ is the zeroth-order momentum flux tensor given by $\Pi_{0}=\rho c_{s}^{2} \delta_{i j}+\rho_{0} u_{i} u_{j} / f_{l}$. In Eq. (A.21), we assume that $\mathbf{B}_{1}=n \mathbf{F}_{1}$. To recover the macroscopic equations, we put $(m / \tau+n)=1$ in Eq. (A. 21). 
Also, the sum of Eq. (A.16) with respect to $\alpha$ yields,

$$
\frac{\partial \rho}{\partial t_{2}}+\delta t\left(\frac{1}{2}-m\right) \nabla \cdot \mathbf{F}_{1}+\frac{1}{2} \delta t \frac{\partial A_{1}}{\partial t_{1}}=A_{2}
$$

By putting $m=1 / 2$ and combining Eqs. (A.20) and (A. 21), one can find the following continuity equation,

$$
\frac{\partial \rho}{\partial t}+\nabla\left(\rho_{0} \mathbf{u}\right)=A-\frac{1}{2} \varepsilon^{2} \delta t \frac{\partial A_{1}}{\partial t_{1}}
$$

Here, the last term is proportional to $\varepsilon^{2} \delta t$ and it can be omitted [23]. Therefore, Eq.(1) can be reproduced by defining $A$ as,

$$
A=-\beta \frac{\partial f_{s}}{\partial t} \quad \text { (A. 24) }
$$

Finally, by taking the moment of Eq. (A. 16), one can obtain,

$$
\frac{\partial\left(\rho_{0} \mathbf{u}\right)}{\partial t_{2}}=\nabla \cdot \Pi_{1}+\nabla \cdot \boldsymbol{\sigma}
$$

where

$$
\begin{aligned}
& \Pi_{1}=\left(\tau-\frac{1}{2}\right) \delta t\left(c_{s}^{2} \rho_{0}\left(\nabla_{i} \mathbf{u}_{j}+\nabla_{j} \mathbf{u}_{i}\right)\right) \\
& \boldsymbol{\sigma}=\left(\tau-\frac{1}{2}\right) \delta t \frac{\mathbf{u F}+\mathbf{F u}}{f_{l}}-\tau \delta t A c_{s}{ }^{2} \mathbf{I}-\tau \delta t \frac{\mathbf{C}}{f_{l}}
\end{aligned}
$$

When $\sigma=0$, the combination of Eqs. (A.21) and (A.25) yields the macroscopic Navier-Stokes Eq. (2) with Eqs. (6) and (9). Therefore, $\mathbf{C}$ should be given as

$$
\mathbf{C}=\left(1-\frac{1}{2 \tau}\right)(\mathbf{u F}+\mathbf{F u})-A f_{l} C_{s}{ }^{2} \mathbf{I}
$$

By substituting Eq. (A. 24), B $=n \mathbf{F}$ with $n=1-1 /(2 \tau)$ and Eq. (A. 28) into Eq. (A. 9), one obtains Eq. (11). 


\section{References}

[1] M. C. Flemings, Our Understanding of Macrosegregation: Past and Present, ISIJ Int. 40 (2000) 833-841.

[2] C. Beckermann, Modelling of macrosegregation: applications and future needs, Int. Mater. Rev. 47 (2002) 243-261.

[3] G. Lesoult, Macrosegregation in steel strands and ingots: Characterisation, formation and consequences, Mater. Sci. Eng. A 413-414 (2005) 19-29.

[4] E. D. Pickering, Macrosegregation in Steel Ingots: The Applicability of Modelling and Characterisation Techniques, ISIJ Int. 53 (2013) 935-949.

[5] W. D. Bennon and F. P. Incropera, A continuum model for momentum, heat and species transport in binary solid-liquid phase change systems-I. Model formulation, Int. J. Heat Mass Transfer 30 (1987) 2161-2170.

[6] J. Ni and C. Beckermann, A volume-averaged two-phase model for transport phenomena during solidification, Metall. Trans. B 22B (1991) 349-361.

[7] M. Wu and A. Ludwig, A three-phase model for mixed columnar-equiaxed solidification, Metall. Mater. Trans. A 37 (2006) 1613-1631.

[8] B. Benzi, S. Succi and M. Vergassola, The lattice Boltzmann equation: theory and applications, Phys. Rep. 222 (1992) 145-197.

[9] S. Chen and G. D. Doolen, LATTICE BOLTZMANN METHOD FOR FLUID FLOWS, Annu. Rev. Fluid Mech. 30 (1998) 329-364.

[10] C. K. Aidun and J. R. Clausen, Lattice-Boltzmann Method for Complex Flows, Annu. Rev. Fluid Mech. 42 (2010) 439-472.

[11] X. He and L. S. Luo, Lattice Boltzmann Model for the Incompressible NavierStokes Equation, J. Stat. Phys. 88 (1997) 927-944.

[12] T. Inamuro, M. Yoshino and F. Ogino, Accuracy of the lattice Boltzmann method for small Knudsen number with finite Reynolds number, Phys. Fluids 9 (1997) 3535-3542.

[13] Z. Guo, B. Shi and N. Wang, Lattice BGK Model for Incompressible NavierStokes Equation, J. Comput. Phys. 165 (2000) 288-306.

[14] Z. Guo and T. S. Zhao, Lattice Boltzmann model for incompressible flows through porous media, Phys. Rev. E 66 (2002) 036304.

[15] M. Ohno and S. Sato, Acceleration of Macrosegregation Simulation Based on Lattice Boltzmann Method, ISIJ Int. 58 (2018) 114-122.

[16] L. Wang, J. Mi and Z. Guo, A modified lattice Bhatnagar-Gross-Krook model for convection heat transfer in porous media, Int. J. Heat Mass Transfer 94 (2016) 
269-291.

[17] T. Sawada, K. Oikawa, K. Anzai, F. Takahashi, K. Kajikawa and H. Yamada, Direct Simulation for Channel Segregation with Using Stabilized SOLA Method, J. JFS 81 (2009) 283-288.

[18] T. Sawada, K. Oikawa, K. Anzai, F. Takahashi, K. Kajikawa and H. Yamada, Three-dimensional Numerical Simulation of Channel Segregation in Directionally Solidified Sn-20 mass\% Bi Ingot, J. JFS 81 (2009) 177-182.

[19] K. Oikawa, N. Hirata and K. Anzai, Numerical Simulation of Effect of Thermo-solutal Flow on Macrosegregation in Continuously Cast Slabs, Tetsu-to-Hagané 103 (2017) 747-754.

[20] A. A. Amsden and F. H. Harlow, A Simplified MAC Technique for Incompressible Fluid Flow Calculations, J. Comput. Phys. 6 (1970) 322-325.

[21] S. V. Patankar: Numerical Heat Transfer and Fluid Flow, Hemisphere, Washington, DC, (1980), 126.

[22] A. Harten, High Resolution Schemes for Hyperbolic Conservation Laws, J. Comput. Phys. 49 (1997) 260-278.

[23] Z. Guo, C. Zheng and B. Shi, Discrete lattice effects on the forcing term in the lattice Boltzmann method, Phys. Rev. E 65 (2002) 046308.

[24] Q. Liu, Y.-L. He, Q. Li and W.-Q. Tao, A multiple-relaxation-time lattice Boltzmann model for convection heat transfer in porous media, Int. J. Heat Mass Transfer 73 (2014) 761-775.

[25] J. Zhang, L. Wang, J. Ouyang, Lattice Boltzmann Model for The Volume-Averaged Navier-Stokes Equations, EPL 107 (2014), 20001.

[26] R. Rojas, T. Takaki and M. Ohno, A phase-field-lattice Boltzmann method for modeling motion and growth of a dendrite for binary alloy solidification in the presence of melt convection, J. Comput. Phys. 298 (2015) 29-40.

[27] T. Takaki, R. Sato, R. Rojas, M. Ohno and Y. Shibuta, Phase-field lattice Boltzmann simulations of multiple dendrite growth with motion, collision, and coalescence and subsequent grain growth, Comput. Mater. Sci. 147 (2018) 124-131.

[28] S. Sakane, T. Takaki, M. Ohno, Y. Shibuta, T. Shimokawabe and T. Aoki, Three-dimensional morphologies of inclined equiaxed dendrites growing under forced convection by phase-field-lattice Boltzmann method, J. Cryst. Growth 483 (2018) 147-155.

[29] Z. Guo, C. Zheng and B. Shi, Discrete lattice effects on the forcing term in the lattice Boltzmann method, Phys. Rev. E 65 (2012) 046308-1-6. 
Table 1. Parameters used for simulation of lateral directional solidification of Sn-Bi alloy [18]

\begin{tabular}{|l|l|}
\hline Initial composition of Bi, $C_{\mathrm{Bi}}{ }^{0}[\mathrm{mass} \%]$ & 10 \\
\hline Density of $\mathrm{Sn}, \rho_{\mathrm{Sn}}\left[\mathrm{Kg} \mathrm{m}^{-3}\right]$ & 7310 \\
\hline Density of $\mathrm{Sn}, \rho_{\mathrm{Bi}}\left[\mathrm{Kg} \mathrm{m}^{-3}\right]$ & 9780 \\
\hline Kinematic viscosity, $v\left[\mathrm{~m}^{2} \mathrm{~s}^{-1}\right]$ & $2.5 \times 10^{-7}$ \\
\hline Thermal conductivity, $\lambda\left[\mathrm{W} \mathrm{m}^{-1} \mathrm{~K}^{-1}\right]$ & 60 \\
\hline Latent heat, $\Delta H\left[\mathrm{~J} \mathrm{Kg}^{-1}\right]$ & 60000 \\
\hline Specific heat, $c_{h}\left[\mathrm{~J} \mathrm{~K}^{-1} \mathrm{Kg}^{-1}\right]$ & 250 \\
\hline Thermal diffusivity, $a_{T}\left[\mathrm{~m}^{2} \mathrm{~s}^{-1}\right]$ & $2.5 \times 10^{-5}$ \\
\hline Heat transfer coefficient, $h\left[\mathrm{~W} \mathrm{~m}^{-2} \mathrm{~K}^{-1}\right]$ & 10000 \\
\hline Thermal expansion coefficient, $\beta_{T}\left[\mathrm{~K}^{-1}\right]$ & $1.0 \times 10^{-4}$ \\
\hline Permeability coefficient, $K_{0}\left[\mathrm{~m}^{2}\right]$ & $1.5 \times 10^{-10}$ \\
\hline Solidification shrinkage rate, $\beta[-]$ & 0 \\
\hline
\end{tabular}

Table 2. Spatial grid and time steps

\begin{tabular}{|l|l|l|l|}
\hline & Case 1 & Case 2 & Case 3 \\
\hline Grid spacing, $\delta x[\mathrm{~mm}]$ & 1.0 & 0.5 & 0.25 \\
\hline Time step, $\delta t[\mathrm{~ms}]$ & 1.0 & 0.5 & 0.25 \\
\hline Total number of grids, $N_{\text {grid }}$ & $64^{2}$ & $128^{2}$ & $256^{2}$ \\
\hline Total time steps, $N_{\text {time }}$ & 1600000 & 3200000 & 6400000 \\
\hline Relaxation time, $\tau$ & 0.51075 & 0.5115 & 0.513 \\
\hline
\end{tabular}


Table 3. Parameters used for simulation of continuously cast steel slab ${ }^{[19]}$

\begin{tabular}{|l|l|}
\hline Initial composition of C, $C_{\mathrm{C}}{ }^{0}[\mathrm{mass} \%]$ & 0.55 \\
\hline Density of $\mathrm{Fe}, \rho_{\mathrm{Fe}}\left[\mathrm{Kg} \mathrm{m}^{-3}\right]$ & 7100 \\
\hline Density of $\mathrm{C}, \rho_{\mathrm{C}}\left[\mathrm{Kg} \mathrm{m}^{-3}\right]$ & 3440 \\
\hline Kinematic viscosity, $v\left[\mathrm{~m}^{2} \mathrm{~s}^{-1}\right]$ & $6.0 \times 10^{-7}$ \\
\hline Thermal conductivity, $\lambda\left[\mathrm{W} \mathrm{m}^{-1} \mathrm{~K}^{-1}\right]$ & 41.86 \\
\hline Latent heat, $\Delta H\left[\mathrm{~J} \mathrm{Kg}^{-1}\right]$ & 251163 \\
\hline Specific heat, $c_{h}\left[\mathrm{~J} \mathrm{~K}^{-1} \mathrm{Kg}^{-1}\right]$ & 837 \\
\hline Thermal diffusivity, $a_{T}\left[\mathrm{~m}^{2} \mathrm{~s}^{-1}\right]$ & $7.1 \times 10^{-6}$ \\
\hline Heat transfer coefficient, $h\left[\mathrm{~W} \mathrm{~m}^{-2} \mathrm{~K}^{-1}\right]$ & $200 *$ \\
\hline Thermal expansion coefficient, $\beta_{T}\left[\mathrm{~K}^{-1}\right]$ & $8.28 \times 10^{-4}$ \\
\hline Secondary dendrite arm spacing, $\lambda_{2}[\mathrm{~m}]$ & $1.7 \times 10^{-4}$ \\
\hline Permeability coefficient, $K_{0}\left[\mathrm{~m}^{2}\right]$ & $5.0 \times 10^{-11}$ \\
\hline Solidification shrinkage rate, $\beta[-]$ & 0.03 \\
\hline
\end{tabular}

* Since the heat transfer coefficient reported in Ref. [19] was quite low, i.e., $0.005 \mathrm{~W} \mathrm{~m}^{-2}$ $\mathrm{K}^{-1}$, we assumed $200 \mathrm{~W} \mathrm{~m} \mathrm{~K}^{-2} \mathrm{~K}^{-1}$ which yields the solidification time quite similar to the one in Ref. [19]. 
(a) $v^{*}=0.15$

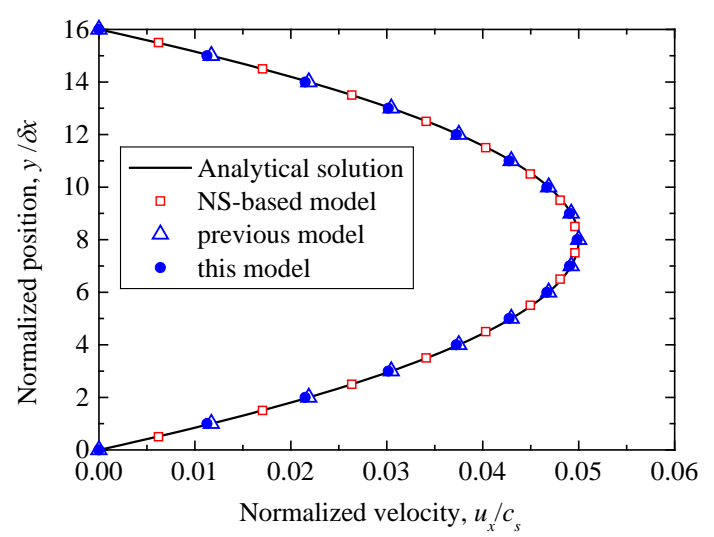

(b) $v^{*}=0.03$

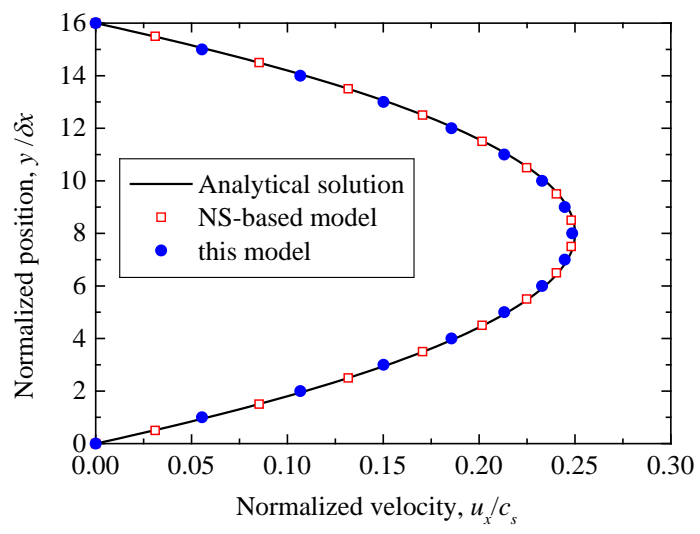

Fig.1. Calculated results of steady-state velocity in fluid systems with (a) $v^{*}=0.15$ and (b) $v^{*}=0.05$ between two parallel planes. In each figure, the solid line represents the analytical solution. Squares and full circles indicate the results of NS-based model and the present LBM-coupled model, respectively. In Fig. 1(a), open circles represent the results of the previous LBM-coupled model [15]. 


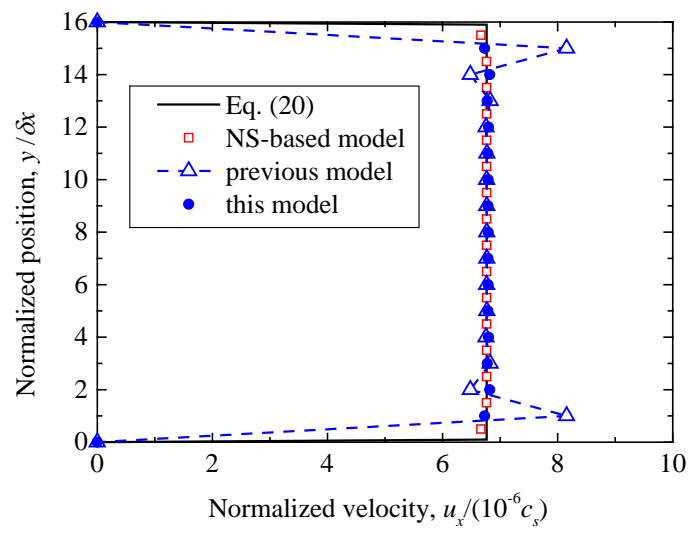

Fig.2. Calculated results of steady-state velocity in a solid and liquid two-phase system with $f_{s}=0.5$ between two parallel planes. The solid line represents the solution of Eq. (20) and red square indicates the result of NS-based model. The open and filled circles represent the results of the previous [15] and present LBM-coupled models. The dashed line with the open circles (the previous LBM-coupled model) is guide to the eyes. 
(a) $f_{\mathrm{s}}$ and $\mathbf{u}$ at $t=450 \mathrm{~s}$

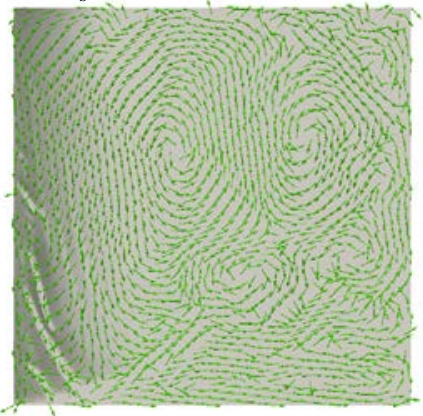

(d) $C_{\text {seg }}$ at $t=450 \mathrm{~s}$

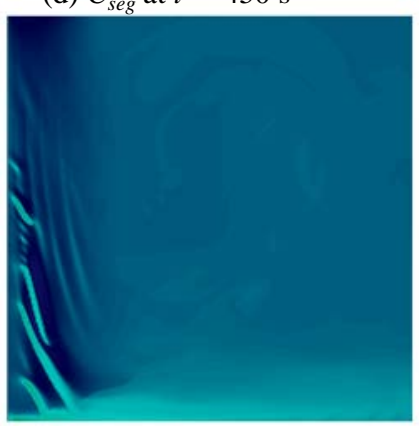

(b) $f_{\mathrm{s}}$ and $\mathbf{u}$ at $t=550 \mathrm{~s}$

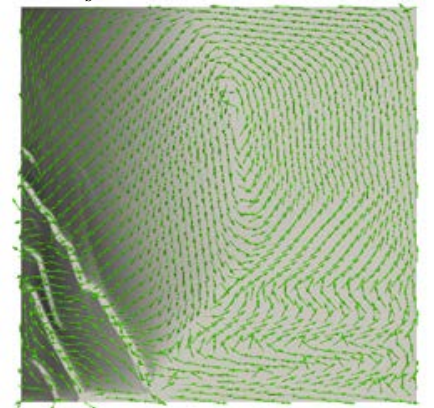

(e) $C_{\text {seg }}$ at $t=550 \mathrm{~s}$

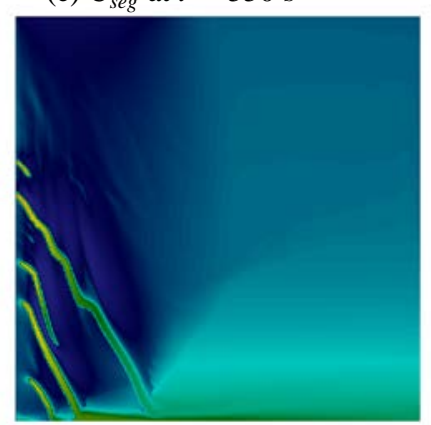

(c) $f_{\mathrm{s}}$ and $\mathbf{u}$ at $t=650 \mathrm{~s}$

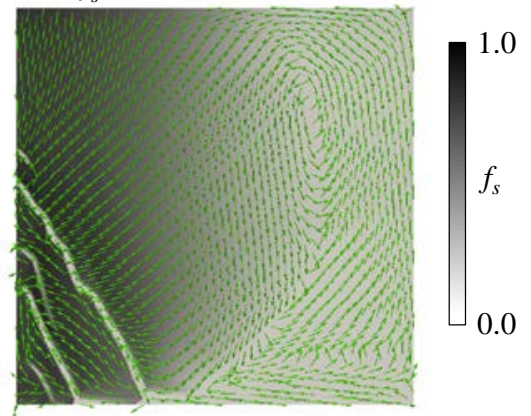

(f) $C_{\text {seq }}$ at $t=650 \mathrm{~s}$

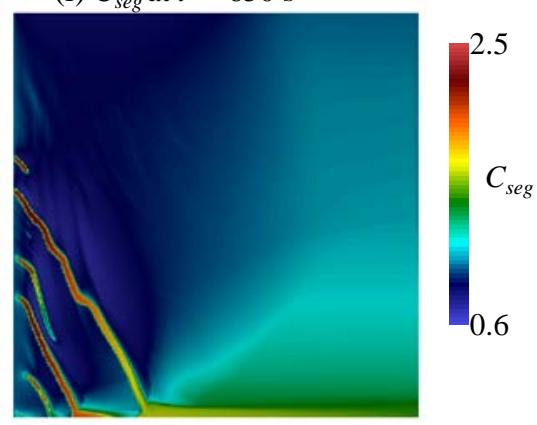

Fig. 3. Results of lateral directional solidification in Sn-10mass\%Bi alloy obtained by the LBM-coupled model with $256^{2}$ grid points. The snapshots in (a-c) show $f_{s}$ (gray scale) and u-field (arrow) at (a) $t=450 \mathrm{~s}$, (b) $550 \mathrm{~s}$ and (c) $650 \mathrm{~s}$, while those in (d-f) represent the segregation ratio at (a) $t=450 \mathrm{~s}$, (b) $550 \mathrm{~s}$ and (c) $650 \mathrm{~s}$. 
(a) LBM-coupled model

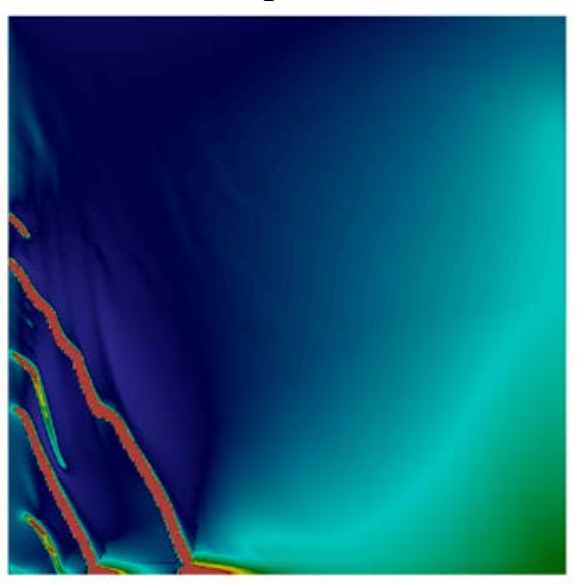

(b) NS-based model

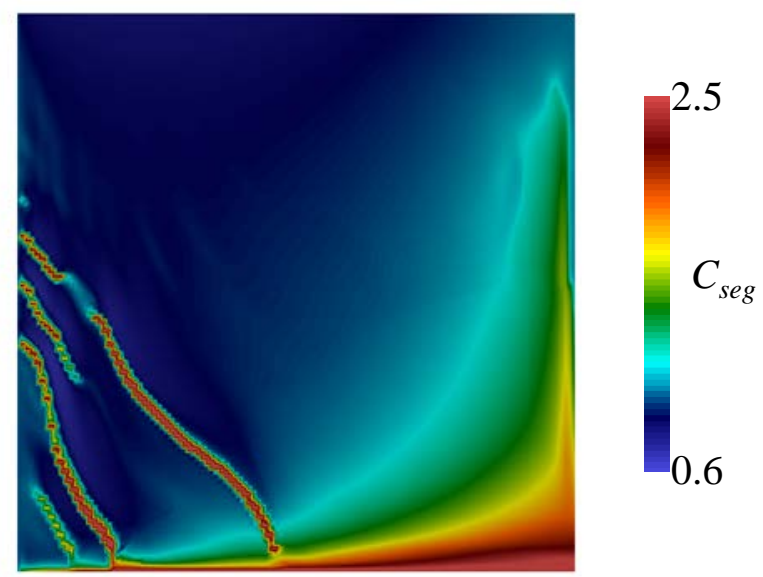

Fig. 4. Segregation patterns at $1600 \mathrm{~s}$ calculated by (a) LBM-coupled model and (b) NS-based model. $128^{2}$ grid points were utilized in both simulations. 


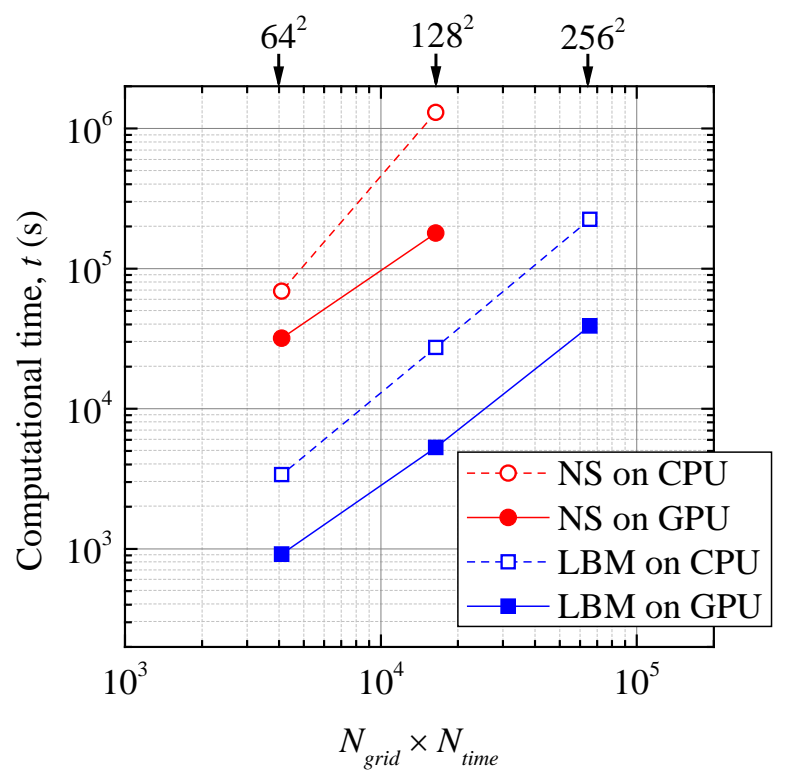

Fig. 5. Computational times of NS-based model (circles) and LBM-coupled model (squares) on CPU (open symbols) and GPU(full symbols). 
(a)

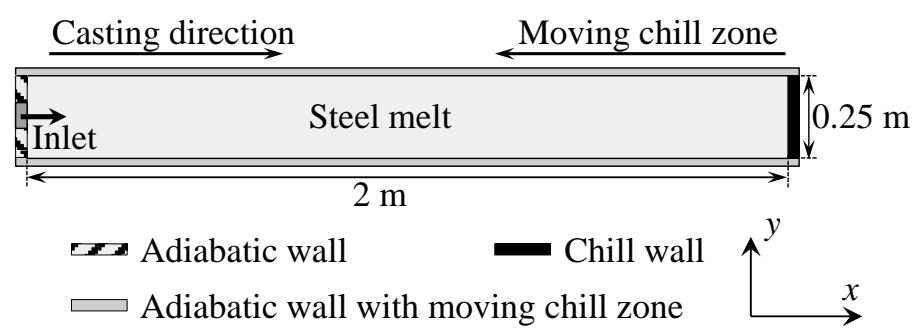

(b)

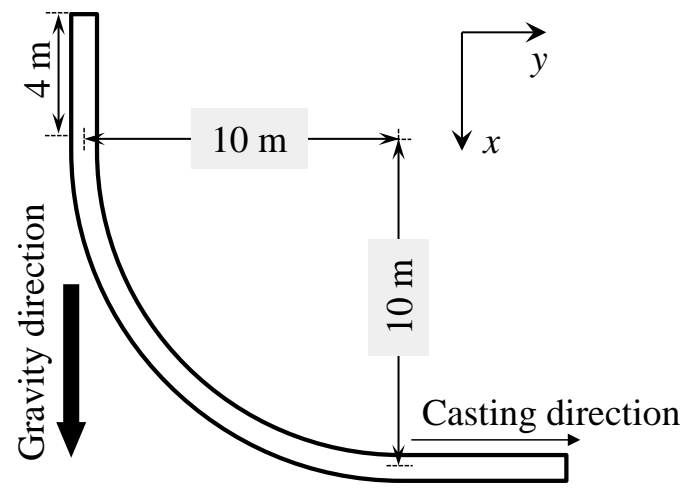

Fig. 6. Schematic illustrations of (a) computational box of continuously cast steel slab and (b) continuous caster [19]. 
(a) $t=400 \mathrm{~s}$

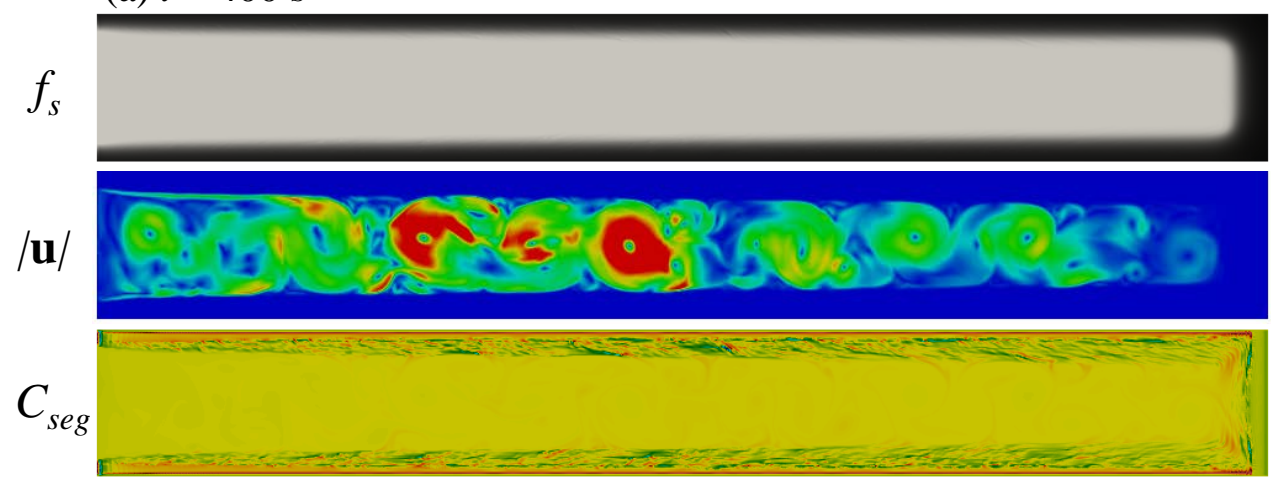

(b) $t=800 \mathrm{~s}$

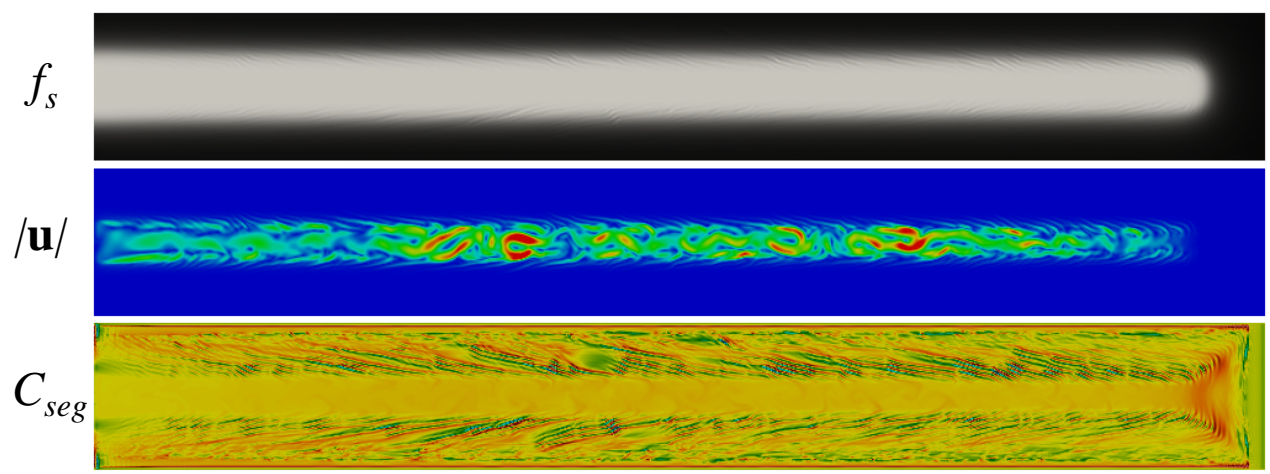

(c) $t=1200 \mathrm{~s}$
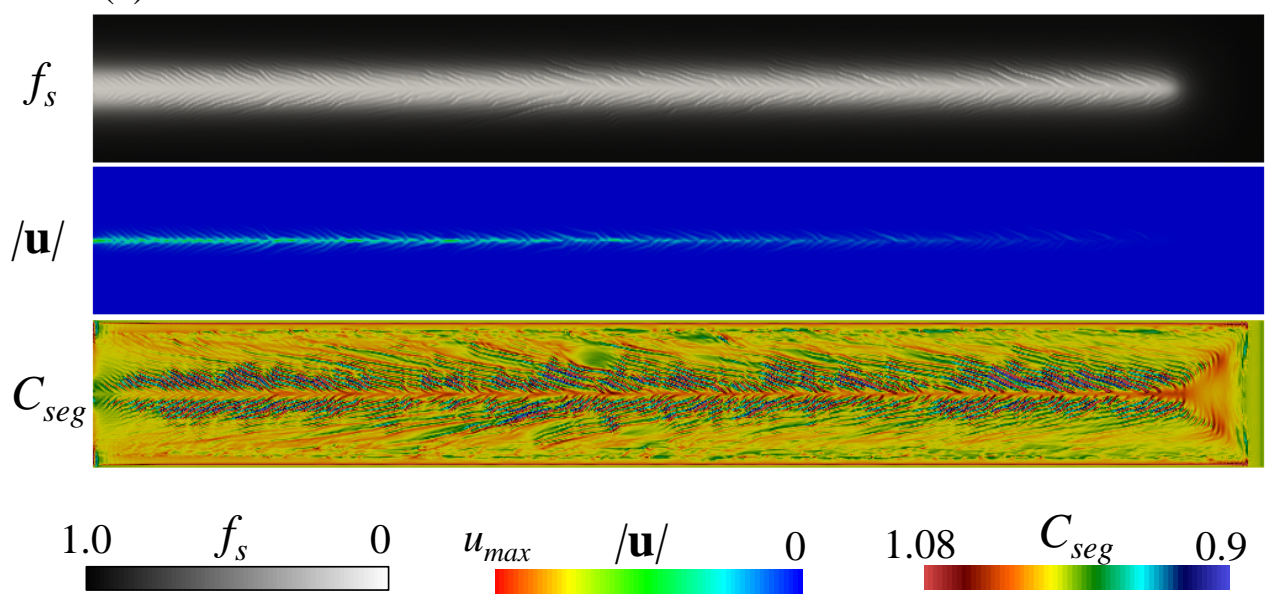

Fig. 7. Simulation results of continuous casting of steel slab at (a) $t=400 \mathrm{~s}$, (b) $t=800 \mathrm{~s}$ and (c) $t=1200 \mathrm{~s}$. In each figure, the upper, middle and lower snapshots represent the spatial distributions of $f_{s},|\mathbf{u}|$ and $C_{\text {seg }}$ of carbon, respectively. The scale bars of these variables are shown at the bottom. $u_{\max }$ in Fig. (a) is $0.01 \mathrm{~m} / \mathrm{s}$, while the one in Figs. (b) and (c) is $0.001 \mathrm{~m} / \mathrm{s}$. 